\title{
A NEW STELLAR CHEMO-KINEMATIC RELATION REVEALS THE MERGER HISTORY OF THE MILKY WAY DISK
}

\author{
I. Minchev ${ }^{1}$, C. Chiappini ${ }^{1}$, M. Martig ${ }^{2}$, M. Steinmetz ${ }^{1}$, R. S. De Jong ${ }^{1}$, C. Boeche ${ }^{3}$, C. Scannapieco ${ }^{1}$, T. Zwitter ${ }^{4,5}$, \\ R. F. G. Wyse ${ }^{6}$, J. J. Binney ${ }^{7}$, J. Bland-Hawthorn ${ }^{8}$, O. Bienaymé ${ }^{9}$, B. Famaey ${ }^{9}$, K. C. Freeman ${ }^{10}$, B. K. Gibson ${ }^{11}$, \\ E. K. Grebel ${ }^{3}$, G. Gilmore ${ }^{12}$, A. Helmi ${ }^{13}$, G. Kordopatis ${ }^{12}$, Y. S. LeE $^{14}$, U. Munari ${ }^{15}$, J. F. Navarro ${ }^{16,23}$, Q. A. Parker ${ }^{17,18,19}$, \\ A. C. Quillen ${ }^{20}$, W. A. Reid ${ }^{17,18}$, A. Siebert ${ }^{9}$, A. Siviero ${ }^{1,21}$, G. Seabroke ${ }^{22}$, F. Watson $^{19}$, and M. Williams ${ }^{1}$ \\ ${ }_{1}^{1}$ Leibniz-Institut für Astrophysik Potsdam (AIP), An der Sternwarte 16, D-14482 Potsdam, Germany \\ ${ }^{2}$ Centre for Astrophysics and Supercomputing, Swinburne University of Technology, P.O. Box 218, Hawthorn, VIC 3122, Australia \\ ${ }^{3}$ Astronomisches Rechen-Institut, Zentrum für Astronomie der Universität Heidelberg, Mönchhofstr. 12-14, D-69120 Heidelberg, Germany \\ ${ }^{4}$ Faculty of Mathematics and Physics, University of Ljubljana, Jadranska 19, 1000 Ljubljana, Slovenia \\ ${ }^{5}$ Center of Excellence SPACE-SI, Askerceva 12, 1000 Ljubljana, Slovenia \\ ${ }^{6}$ Department of Physics and Astronomy, Johns Hopkins University, 3400 North Charles Street, Baltimore, MD 21218, USA \\ ${ }^{7}$ Rudolf Peierls Centre for Theoretical Physics, Keble Road, Oxford OX1 3NP, UK \\ ${ }^{8}$ Sydney Institute for Astronomy, School of Physics, University of Sydney, Sydney, NSW 2006, Australia \\ ${ }^{9}$ CNRS, Observatoire Astronomique, Université de Strasbourg, 11 rue de l'Université, F-67000 Strasbourg, France \\ ${ }^{10}$ Australian National University, Canberra, ACT 0200, Australia \\ ${ }^{11}$ Jeremiah Horrocks Institute, University of Central Lancashire, Preston PR1 2HE, UK \\ 12 Institute of Astronomy, University of Cambridge, Madingley Road, Cambridge CB3 OHA, UK \\ ${ }^{13}$ Kapteyn Astronomical Institute, University of Groningen, P.O. Box 800, 9700-AV Groningen, The Netherlands \\ ${ }^{14}$ Department of Astronomy, New Mexico State University, Las Cruces, NM 88003, USA \\ ${ }^{15}$ INAF National Institute of Astrophysics, Astronomical Institute of Padova, I-36012 Asiago (VI), Italy \\ ${ }^{16}$ University of Victoria, P.O. Box 3055, Station CSC, Victoria, BC V8W 3P6, Canada \\ ${ }^{17}$ Department of Physics and Astronomy, Macquarie University, Sydney, NSW 2109, Australia \\ ${ }^{18}$ Research Centre for Astronomy, Astrophysics and Astrophotonics, Macquarie University, Sydney, NSW 2109, Australia \\ ${ }^{19}$ Australian Astronomical Observatory, P.O. Box 915, North Ryde, NSW 1670, Australia \\ ${ }^{20}$ Department of Physics and Astronomy, University of Rochester, Rochester, NY 14627, USA \\ ${ }^{21}$ Department of Physics and Astronomy, Padova University, Vicolo dell Osservatorio 2, I-35122 Padova, Italy \\ ${ }^{22}$ Mullard Space Science Laboratory, University College London, Holmbury St. Mary, Dorking RH5 6NT, UK \\ Received 2013 October 18; accepted 2013 December 23; published 2014 January 6
}

\begin{abstract}
The velocity dispersions of stars near the Sun are known to increase with stellar age, but age can be difficult to determine, so a proxy like the abundance of $\alpha$ elements (e.g., $\mathrm{Mg}$ ) with respect to iron, $[\alpha / \mathrm{Fe}]$, is used. Here we report an unexpected behavior found in the velocity dispersion of a sample of giant stars from the Radial Velocity Experiment survey with high-quality chemical and kinematic information, in that it decreases strongly for stars with $[\mathrm{Mg} / \mathrm{Fe}]>0.4$ dex (i.e., those that formed in the first gigayear of the Galaxy's life). These findings can be explained by perturbations from massive mergers in the early universe, which have affected the outer parts of the disk more strongly, and the subsequent radial migration of stars with cooler kinematics from the inner disk. Similar reversed trends in velocity dispersion are also found for different metallicity subpopulations. Our results suggest that the Milky Way disk merger history can be recovered by relating the observed chemo-kinematic relations to the properties of past merger events.
\end{abstract}

Key words: Galaxy: abundances - Galaxy: disk - Galaxy: evolution - Galaxy: formation - Galaxy: kinematics and dynamics - solar neighborhood

Online-only material: color figures

\section{INTRODUCTION}

Understanding galaxy formation and evolution is one of the central goals of contemporary astronomy and cosmology. High-redshift observations provide insight into the evolution of global galaxy properties, but are fundamentally limited in probing the internal kinematics and chemistry on sub-galactic scales. The Milky Way is the only galaxy within which we can obtain information at the level of detail required to understand these processes. This realization is manifested in the number of on-going and planned spectroscopic Milky Way surveys, such as the Radial Velocity Experiment (RAVE) (Steinmetz et al. 2006), SEGUE (Yanny et al. 2009), APOGEE (Majewski et al. 2010), HERMES (Freeman 2010), Gaia-ESO (Gilmore

\footnotetext{
${ }^{23}$ Senior CIfAR Fellow.
}

et al. 2012), Gaia (Perryman et al. 2001), and 4MOST (de Jong et al. 2012), which aim to obtain kinematic and chemical information for a large number of stars. Despite the increasing amount of observational data, to date we have lacked the means to discriminate among different thick-disk formation scenarios and to understand the disk merger history in general. Here we fill this gap.

Stars near the Sun have long been known to follow an age-velocity relation, where velocity dispersions increase with age (Wielen 1977). Due to the lack of good age estimates, the shape of the age-velocity relation has been a matter of debate (Freeman 1991; Binney et al. 2000; Seabroke \& Gilmore 2007). Alternatively, the $[\alpha / \mathrm{Fe}]$ ratios, such as $[\mathrm{Mg} / \mathrm{Fe}]$ can be used to identify the oldest stars because the interstellar medium is quickly enriched in elements formed by $\alpha$-capture in short-lived massive stars, while there is a delay before iron-peak elements 

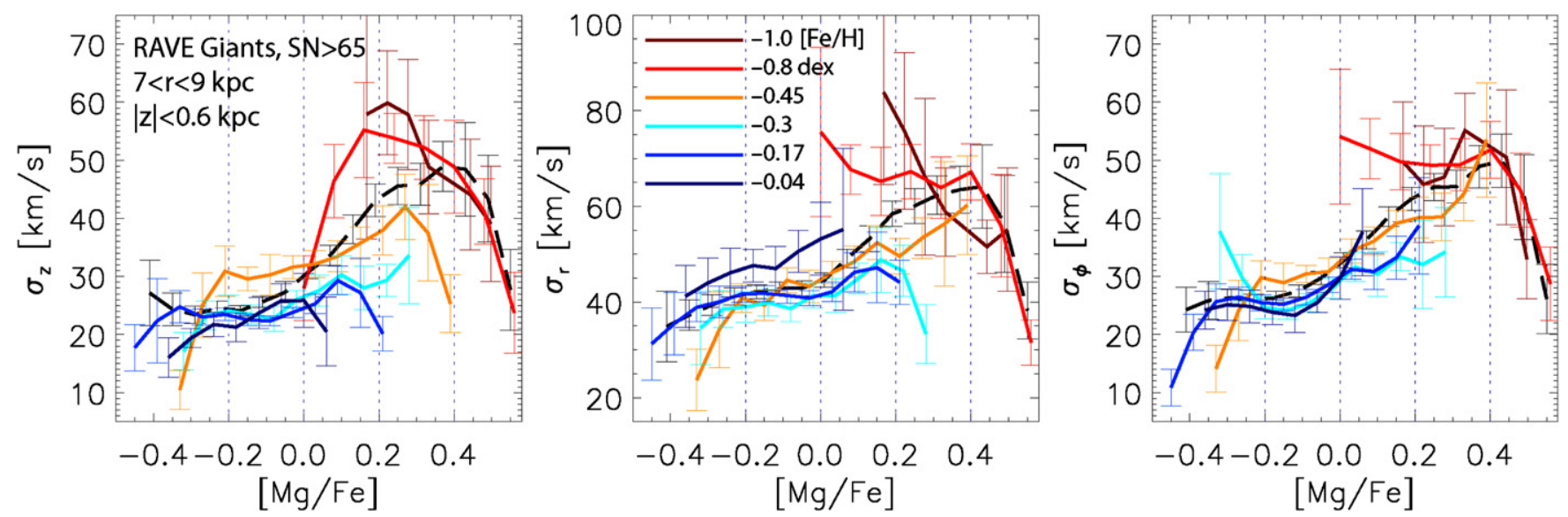

Figure 1. Left: vertical velocity dispersion $\sigma_{z}$, as a function of $[\mathrm{Mg} / \mathrm{Fe}]$ ratios for RAVE giants. The black dashed curve shows the total sample. The color-coded curves present subpopulations grouped by common median metallicity as indicated in the middle panel, with resulting mean values $[\mathrm{Fe} / \mathrm{H}]=-1.05,-0.85,-0.45,-0.3,-0.1,+0.125$ dex. The error for each $[\mathrm{Mg} / \mathrm{Fe}]$ bin is estimated as the two standard deviations of 1000 realizations in a bootstrapping calculation. The number of stars in the three highest $[\mathrm{Mg} / \mathrm{Fe}]$ bins are 43,25 , and 11 , respectively. Because variation in both chemistry and kinematics is expected with changes in position in the galactic disk, we constrain our sample to galactocentric distances in the range $7<r<9 \mathrm{kpc}$ and consider a maximum vertical height above and below the disk plane $|z|=0.6 \mathrm{kpc}$, where $r$ and $z$ are the radial and vertical coordinates in a cylindrical system. Middle: same as on the left, but for the radial velocity dispersion $\sigma_{r}$. Right: same as on the left and middle, but for the azimuthal velocity dispersion $\sigma_{\phi}$. Similar reversal in the velocity dispersion trends at $[\mathrm{Mg} / \mathrm{Fe}]>0.4 \mathrm{dex}$ is found for all velocity components.

(A color version of this figure is available in the online journal.)

are produced in abundance by thermonuclear supernovae (SNIa; Matteucci 2012; Haywood et al. 2013).

Studying the SEGUE G-dwarf sample, Bovy et al. (2012) and Liu \& van de Ven (2012) have recently argued that the stellar vertical velocity dispersion increases for populations of decreasing metallicity and increasing $[\alpha / \mathrm{Fe}]$, but this relation has been somewhat unclear at the low-metallicity end. We reexamine the $\sigma_{z}-[\mathrm{Fe} / \mathrm{H}]-[\alpha / \mathrm{Fe}]$ connection by presenting it in a different way compared to earlier works: instead of color maps, here we overlay curves of velocity dispersion with $[\mathrm{Mg} / \mathrm{Fe}]$ for only six different metallicity bins, allowing for better statistics in the low-metallicity regime where the number of stars becomes small.

\section{RESULTS}

\subsection{Chemo-Kinematic Relation in RAVE}

We study data from RAVE (Steinmetz et al. 2006; Zwitter et al. 2008; Siebert et al. 2011; Kordopatis et al. 2013), a magnitude-limited survey of stars randomly selected in the $9<I<12$ magnitude range which is currently the largest spectroscopic sample of stars in the Milky Way for which individual elemental abundances are available (Boeche et al. 2011).

For this work we selected a RAVE sample of giant stars which is close to a random magnitude-limited sample. We excluded giants with $\log g<0.5$ to avoid any possible effects due to the boundaries of the learning grid used for the automated parameterization, and considered stars in the temperature range $4000<T_{\text {eff }}<5500$ (thus avoiding horizontal branch stars; see Boeche et al. 2013).

The estimated total measurement uncertainties (internal plus external) of the new RAVE-DR4 pipeline for giants ( $\log g<$ $3.5 \mathrm{dex})$ with a signal-to-noise ratio $(\mathrm{S} / \mathrm{N})>50$ in the temperature and metallicity range we consider in this work $(-1.5<$ $[\mathrm{Fe} / \mathrm{H}]<0.5$ ) are (see Table 2 in Kordopatis et al. 2013) $\sim 100 \mathrm{~K}$ in $T_{\text {eff }}, \sim 0.3$ dex for $\log g$, and $\sim 0.1$ for the metallicity $[\mathrm{M} / \mathrm{H}]$. The final uncertainties in $[\mathrm{Fe} / \mathrm{H}]$ and $[\mathrm{Mg} / \mathrm{Fe}]$ computed by the chemical pipeline are $\sim 0.1$ and 0.15 dex, respectively.
To further decrease these, we select a sample of giants with $\mathrm{S} / \mathrm{N}>65$, resulting in 4755 stars with high-quality chemistry and kinematics. The mean uncertainties in galactocentric velocities are $10-15 \mathrm{~km} \mathrm{~s}^{-1}$.

In the left panel of Figure 1, the black-dashed curve shows the variation with $[\mathrm{Mg} / \mathrm{Fe}]$ of the vertical velocity dispersion $\sigma_{z}$. Contrary to the expected monotone increase of $\sigma_{z}$ with $\alpha$-enhancement, we see a strong inversion of this trend at $[\mathrm{Mg} / \mathrm{Fe}]>0.4$ dex. This is unexpected because stars with higher $[\alpha / \mathrm{Fe}]$ ratios, at a given radius, should be older and thus should possess larger random energies. The six metallicity $([\mathrm{Fe} / \mathrm{H}])$ bins, shown by color-coded curves, allow us to probe deeper. We see that for $[\mathrm{Fe} / \mathrm{H}]>-0.4, \sigma_{z}$ is independent of $[\mathrm{Fe} / \mathrm{H}]$ and a slowly increasing function of $[\mathrm{Mg} / \mathrm{Fe}]$, but that for more metal-poor stars $\sigma_{z}$ increases with decreasing $[\mathrm{Fe} / \mathrm{H}]$. An interesting observation is the distinct decrease in $\sigma_{z}$ at the high- $[\mathrm{Mg} / \mathrm{Fe}]$ end of all but one $[\mathrm{Fe} / \mathrm{H}]$ subpopulation, with the $\sigma_{z}$-maximum shifting to lower $[\mathrm{Mg} / \mathrm{Fe}]$ for bins of increasing metallicity. For all velocity components, the most metal-poor, $\alpha$-rich stars (which must be the oldest) have velocity dispersions comparable to those of the most metal-rich (youngest) populations. In Section 3 we show that this puzzling behavior can be explained by radial migration of old cold stellar samples from the inner disk.

\subsection{Comparison to SEGUE G-dwarfs}

The results we obtained here with RAVE data should be seen in other ongoing/near-future galactic surveys. We check this by studying the currently available SEGUE G-dwarf sample described by Lee et al. (2011), by considering stars with $\mathrm{S} / \mathrm{N}>30$. Due to the lack of individual chemical element measurements in SEGUE, we use their $[\alpha / \mathrm{Fe}]$, which is the average of several $\alpha$-elements. Because of the low number of stars at $|z|<0.5 \mathrm{kpc}$ in SEGUE, a maximum distance above and below the disk plane of $|z|=1 \mathrm{kpc}$ is used (instead of $|z|=0.6 \mathrm{kpc}$ ). This results in a sample comprising $\sim 10,300$ stars. Figure 2 presents the same information as Figure 1, but for SEGUE. We recover the expected strong decline in the vertical 

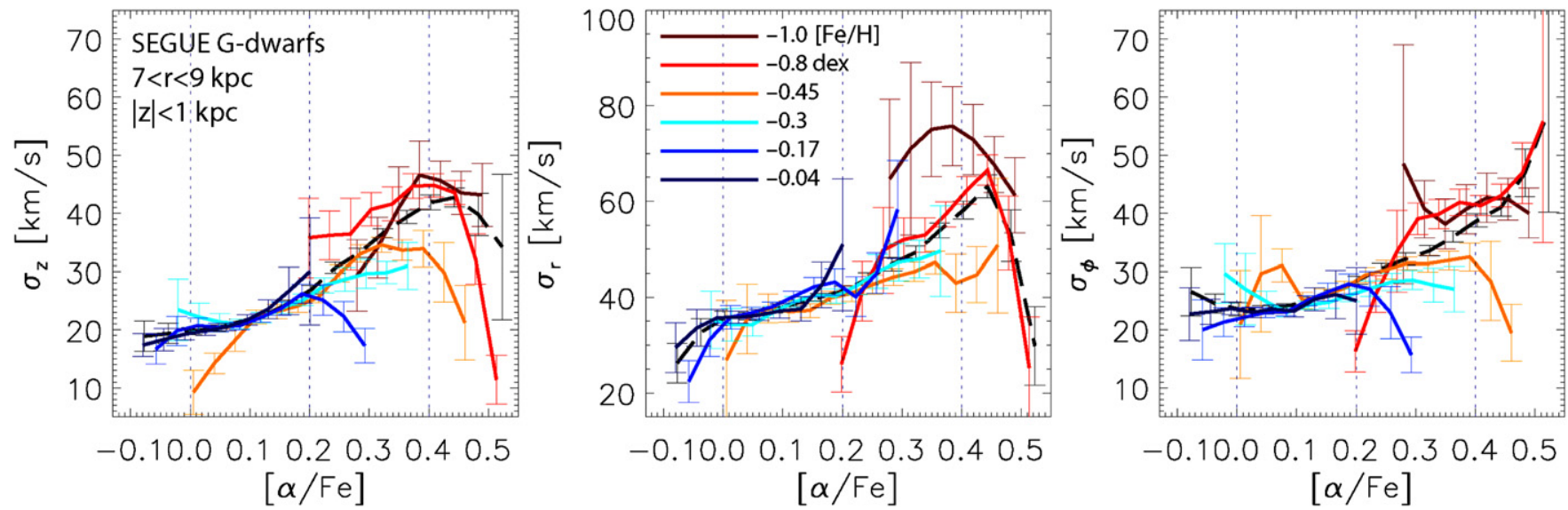

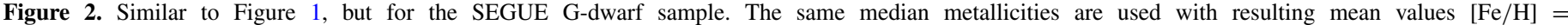

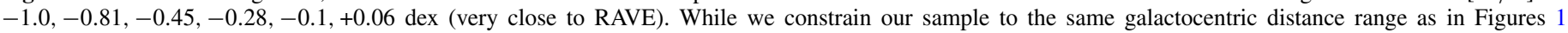

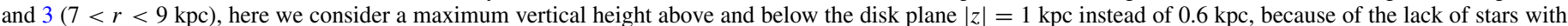
$|z|<0.5 \mathrm{kpc}$ in SEGUE.

(A color version of this figure is available in the online journal.)
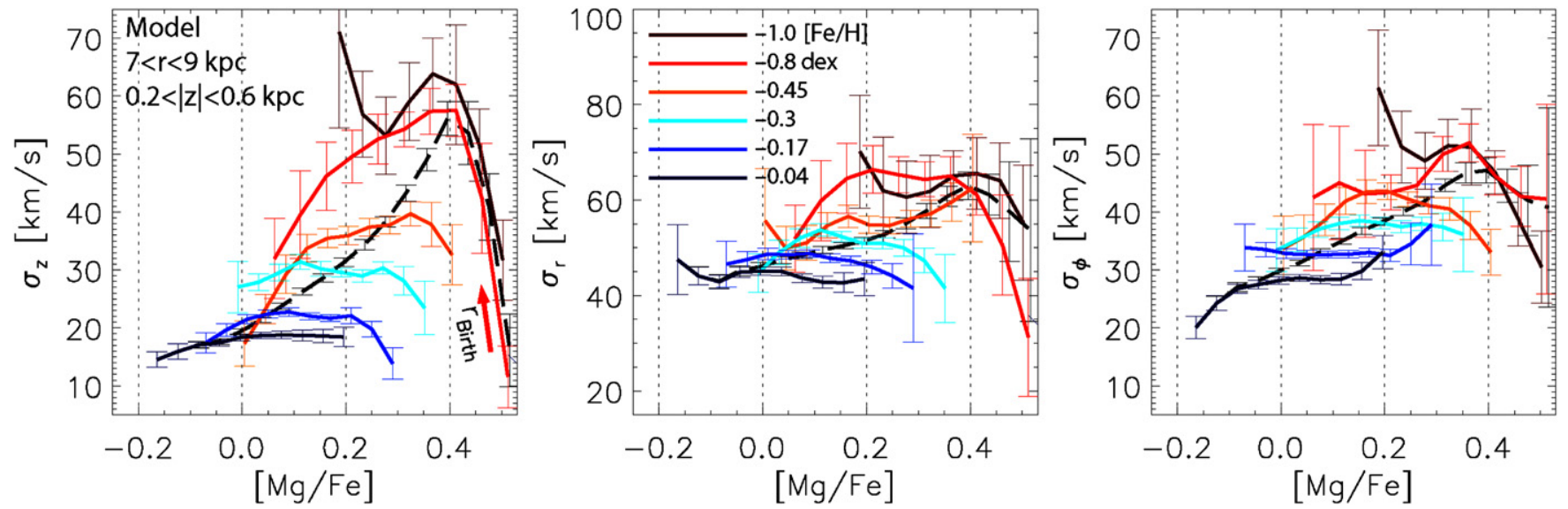

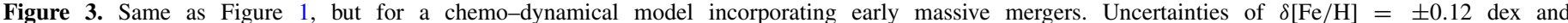

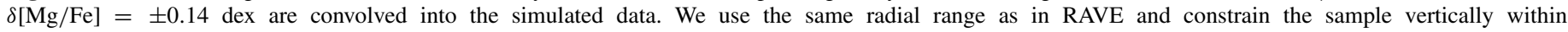

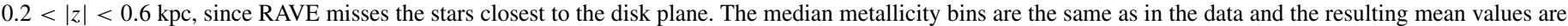

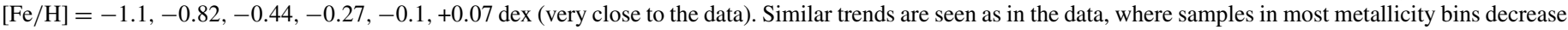
their velocity dispersions at the high- $[\mathrm{Mg} / \mathrm{Fe}]$ end.

(A color version of this figure is available in the online journal.)

and radial velocity dispersions, $\sigma_{z}$ and $\sigma_{r}$, for the most metalpoor samples. While no reversal is found in the dispersion of the azimuthal velocity component, $\sigma_{\phi}$, for the total sample, the expected trend is seen for the intermediate metallicity sub-populations (blue and orange curves) and hinted at in the most metal-poor bin (maroon). This assures us that our findings are not only pertinent to RAVE data, but should provide a general result.

We note that the same trend is hinted at in Figure 2 by Bovy et al. (2012), but is less clear, possibly due to the cut of $S / N>$ 15 , larger $[\alpha / \mathrm{Fe}]$ bins, and a larger disk coverage more prone to erase structure that may vary spatially.

\section{INTERPRETATION}

The highest velocity dispersions $\left(\sigma_{z}, \sigma_{\phi}>40 \mathrm{~km} \mathrm{~s}^{-1}\right.$ and $\sigma_{r}>60 \mathrm{~km} \mathrm{~s}^{-1}$ ) achieved by the lowest three metallicity bins are too large to be accounted for by internal disk evolution processes, such as scattering by giant molecular clouds (Lacey 1984), the spiral arms (Jenkins 1992), or the galactic bar (Minchev et al. 2012a, 2012b). On the other hand, perturbations by mergers at the early times of disk formation (Wyse 2001; Villalobos \& Helmi 2008; Quinn et al. 1993), clumpy disk instabilities (Bournaud et al. 2009), stars born hot at high redshift (Brook et al. 2005, 2012), and/or accreted stellar populations (Abadi et al. 2003; Meza et al. 2005) may be plausible explanations.

We test the possibility that mergers of decreasing satellite-disk-mass ratios and frequency (including stars born hot at high redshift) have caused the trends we find in RAVE giant stars, by using simulated data from a chemo-dynamical model (Minchev et al. 2013) built by the fusion between a high-resolution galaxy simulation in the cosmological context with Milky Way characteristics and a detailed chemical evolution model. A strong perturbation from the last massive merger (1:5 disk mass ratio 8-9 Gyr before present) plays an important role in the disk evolution. Due to its in-plane orbit (inclination less than $45^{\circ}$ ), this event drives strong radial migration by triggering a spiral structure. Similar, but less intense events occur throughout the disk evolution.

Figure 3 presents the same information as Figures 1 and 2, but for the model. A very good agreement is found between the 


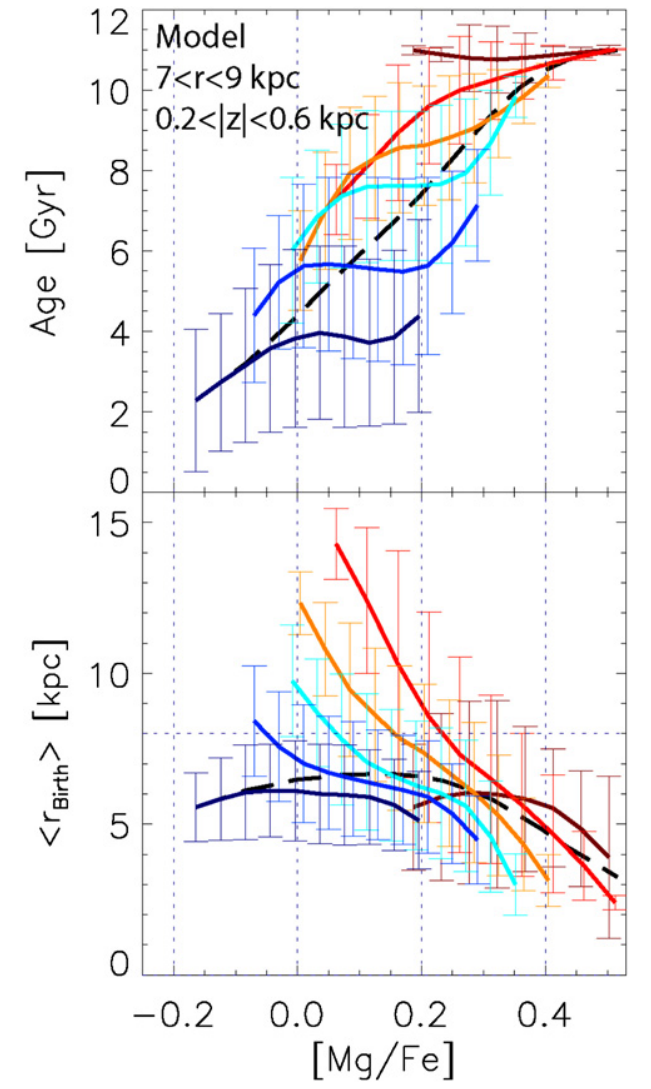

Figure 4. Origin of stars currently in the solar neighborhood. Top: mean age as a function of $[\mathrm{Mg} / \mathrm{Fe}]$ in the simulated solar neighborhood for the same metallicity bins used for the data and model. An almost linear relation between age and $[\mathrm{Mg} / \mathrm{Fe}]$ is found up to $[\mathrm{Mg} / \mathrm{Fe}]=0.4$ dex. However, this is not the case for a given sub-sample, where the mean age is mostly constant and decreasing/increasing at the low/high- $[\mathrm{Mg} / \mathrm{Fe}]$ ends, respectively. Error bars of two standard deviations show the scatter around the mean. Bottom: mean birth-radius, $\left\langle r_{\mathrm{Birth}}\right\rangle$, as a function of $[\mathrm{Mg} / \mathrm{Fe}]$. The dotted horizontal line at $8 \mathrm{kpc}$ indicates the solar radius. The spread in birth radius decreases as more metal-rich (younger) populations are considered, as expected. The inversion in the velocity dispersion trends at $[\mathrm{Mg} / \mathrm{Fe}]>0.4$ dex results from stars born at mean $\left\langle r_{\text {Birth }}\right\rangle<5-6 \mathrm{kpc}$. Note that the velocity dispersion decline at the high- $[\mathrm{Mg} / \mathrm{Fe}]$ end of each metallicity sub-sample is also caused by stars with older mean ages born at progressively smaller radii.

(A color version of this figure is available in the online journal.)

data and model for the velocity dispersion trends of different metallicity subpopulations.

The stars with the highest $[\alpha / \mathrm{Fe}]$-ratios and lowest $[\mathrm{Fe} / \mathrm{H}]$ form in the inner disk at the onset of disk formation in an insideout disk formation scenario. Radial migration can then bring these to the solar vicinity, contaminating the locally evolved sample. Because of the very fast initial chemical evolution, stars with $[\mathrm{Mg} / \mathrm{Fe}]>0.25$ dex and $[\mathrm{Fe} / \mathrm{H}]<-0.5$ dex have approximately the same age, as shown in Figure 4, top. These stars, being the oldest disk population, have the longest time available for migration to the solar vicinity. However, because migration efficiency decreases with increasing velocity dispersion, stars with cold kinematics would be affected the most (Sellwood \& Binney 2002). This is the case for the stars in the metallicity bin with median $[\mathrm{Fe} / \mathrm{H}]=-0.8$ dex (red curve), which are seen to migrate the largest distances (Figure 4, bottom).

\subsection{Radial Migration Cools the Disk During Mergers}

Figures 3 and 4 showed that the decline in velocity dispersion for stars with the highest $[\alpha / \mathrm{Fe}]$ ratios in RAVE and SEGUE

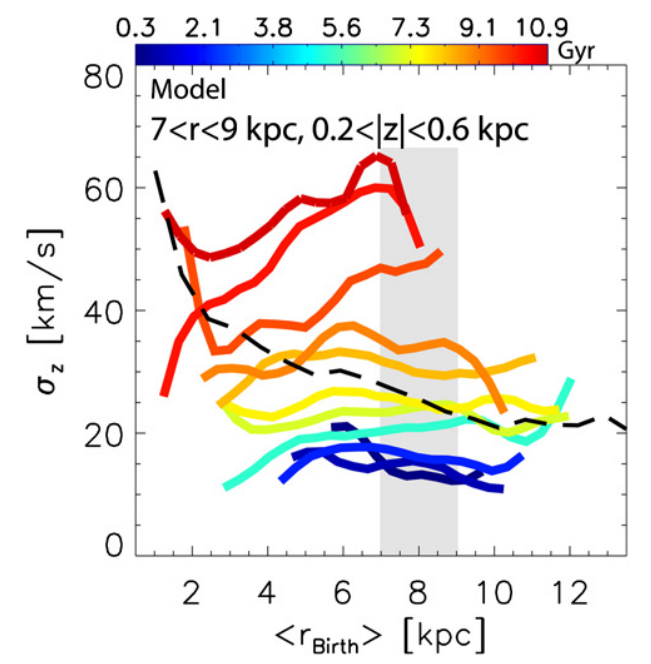

Figure 5. Vertical velocity dispersion, $\sigma_{z}$, as a function of mean birth radius, $\left\langle r_{\text {Birth }}\right\rangle$, for the simulated solar neighborhood sample used in the previous figures. The black-dashed line shows the total population. Different colors correspond to different age groups. The decreasing range in $\left\langle r_{\text {Birth }}\right\rangle$ with age is related to the decreasing time available for radial migration. For the oldest samples stars arrive at the solar vicinity much cooler than stars born in situ, due to the stronger effect of mergers on the outer disk and the decreasing probability of migration with increasing velocity dispersion. The coolest/oldest samples arriving from the innermost disk are the equivalent to the most metal-poor, $[\alpha / \mathrm{Fe}]$-rich stars in RAVE, SEGUE, and the chemo-dynamical model. The large positive gradient found for old populations (red colors) turns into a negative slope for the youngest samples (dark blue), indicating a quiescent regime, where stars arriving from the inner disk slightly heat the local velocity distribution.

(A color version of this figure is available in the online journal.)

data can be interpreted as the effect of stars born in the innermost disk. For this to occur, some of the oldest disk stars must have migrated from the inner disk to the solar neighborhood, while remaining on near-circular orbits, significantly cooler than coeval samples born at progressively larger radii.

In Figure 5, we now check whether this is indeed the case, using the same simulated sample as in all previous figures. The black-dashed curve shows that, for the total sample, the final vertical velocity dispersion, $\sigma_{z}$, increases with decreasing mean birth radius, $\left\langle r_{\text {Birth }}\right\rangle$. Naively, this could be interpreted as evidence that the local disk heats as a result of the migration. However, by decomposing this sample into mono-age populations, we find that the continuous increase in $\sigma_{z}$ with decreasing $\left\langle r_{\text {Birth }}\right\rangle$ is caused by the growing fraction of younger stars born near their current locations, i.e., the solar circle. The fact that stars migrating from the inner disk are generally hotter is related to their older ages, allowing them to be exposed to perturbations causing both heating (Wielen 1977) and migration (Sellwood $\&$ Binney 2002). However, for most coeval populations (color curves) there exists the general trend of $\sigma_{z}$ decreasing with decreasing $\left\langle r_{\text {Birth }}\right\rangle$, which becomes more important for older samples. For the next-to-oldest population, stars originating near the galactic center arrive at the solar vicinity colder by $35 \mathrm{~km} \mathrm{~s}^{-1}$ compared to those born near the Sun. It is clearly seen that stars arriving from the inner disk can be both cooler and older than a locally born sample, which explains the observations.

Figure 5 illustrates that cooler/warmer populations can arrive from the inner/outer disk during mergers, but this trend reverses as external perturbations become unimportant. Therefore, there must exist a critical time at which the disk enters from the first regime into the second. Figure 5 shows that in our model this reversal occurs at $\sim$ age $<1.5 \mathrm{Gyr}$ (dark blue lines). Future work can try to relate this to observations using chemical information. 


\subsection{Implications for the Milky Way Disk Merger History}

From the above discussion it follows that the decrease in the velocity dispersion for the highest- $[\mathrm{Mg} / \mathrm{Fe}]$ stars in the model is a sign that they have arrived in the solar vicinity kinematically cooler than the locally born coeval population. This conclusion is remarkable, as stars migrating outward due to internal instabilities alone are expected to heat the disk (Loebman et al. 2011; Schönrich \& Binney 2009; even if only weakly-Minchev et al. 2012b). The opposite behavior we find here must be related to the presence of mergers in our simulation. Indeed, Figure 4 shows that metal-poor stars with $[\mathrm{Mg} / \mathrm{Fe}]>0.4 \mathrm{dex}$ (which cause the decline in velocity dispersion for the model, the red curves in Figure 3) arrive from progressively smaller galactic radii, while having approximately the same age. Similarly to the most metal-poor stars, the inversion in the $\sigma_{z}-[\mathrm{Mg} / \mathrm{Fe}]$ relation for the other $[\mathrm{Fe} / \mathrm{H}]$ subpopulation can be related to mergers of diminishing strength perturbing the Milky Way disk throughout its lifetime. Within this interpretation, the properties of these events can be recovered in a differential study. For example, satellite-disk-mass ratios and orbital parameters can be related to the mean velocity dispersions of metallicity bins and the inversion in the velocity dispersion profiles as functions of $[\mathrm{Mg} / \mathrm{Fe}]$, for each velocity component.

\section{DISCUSSION}

In this work we presented a new chemo-kinematic relation in RAVE giants, where a decline in the velocity dispersion was found for stars with $[\mathrm{Mg} / \mathrm{Fe}]>0.4 \mathrm{dex}$, as well as for samples in narrow ranges of $[\mathrm{Fe} / \mathrm{H}]$. We verified that another large data set—-the SEGUE G-dwarfs-shows similar relations. By comparison with a chemo-dynamical model, we explained these results as the stronger effect of mergers on the outer parts of disks and the subsequent radial migration of older stellar populations with cooler kinematics born in the inner disk.

This interpretation of our results - that we see the effect of satellite perturbations on the disk-is not strongly model dependent because (1) mergers are expected to always affect the outer disk more significantly (Bournaud et al. 2009), (2) a general prediction of inside-out-formation chemical evolution models is a fast decrease in $[\alpha / \mathrm{Fe}]$ with increasing radius for stars in a narrow metallicity range at high redshift (Matteucci 2012), and (3) radial migration has been firmly accepted to be an inseparable part of disk evolution in numerical simulations (Sellwood \& Binney 2002; Minchev \& Famaey 2010; Roškar et al. 2012). It may be possible that debris accreted from a disrupted galaxy (Abadi et al. 2003; Meza et al. 2005), which should possess the highest velocity dispersions in the disk, had just the right chemistry to create the maximum we observe at $[\mathrm{Mg} / \mathrm{Fe}]=0.4 \mathrm{dex}$, although, to explain the $\sigma_{z}$ maxima of all $[\mathrm{Fe} / \mathrm{H}]$ bins, this interpretation requires a series of mergers of exactly the right increasing metallicity. Furthermore, accretion would not explain the exceedingly low velocity dispersion values for the most $\alpha$-rich stars, which must be linked to radial migration of stars born in the inner disk. The gas-rich turbulent clumpy-disk formation scenario for the thick disk may also provide a viable explanation but in conjunction with perturbations from mergers (making it similar to our model and Brook et al. 2012) necessary to produce the observed decline in velocity dispersion at the high- $[\alpha / \mathrm{Fe}]$ end of each metallicity subpopulation. Finally, a quiescent evolution scenario (e.g., Schönrich \& Binney 2009), where the Milky Way thick disk formed only by heating an initially thin disk through internal evolution processes, is unfeasible, given that in that case stars arriving from the inner disk should be slightly hotter than the locally born population (Loebman et al. 2011; Minchev et al. $2012 b)$, i.e., the contrary to what the observations suggest.

The stars with the lowest $[\mathrm{Fe} / \mathrm{H}]$ and highest $[\mathrm{Mg} / \mathrm{Fe}]$ ratios identified in this work possess the chemistry and kinematics which allow them to be associated with the oldest Milky Way population born in the bar/bulge region. Although efforts are currently being made to look for these in the inner Galaxy, we have shown that, thanks to radial migration, one can also study them here in the solar vicinity.

While the Milky Way has been seen as an unusually quiet galaxy in view of the predictions of the $\Lambda$ CDM theory (Hammer et al. 2007), the results of this work suggests that, in addition to being important for the formation of the thick disk (e.g., Wyse 2001; Minchev et al. 2013), satellite-disk encounters of decreasing intensity were at play throughout its evolution.

To secure the conclusions of this work, follow-up highresolution spectroscopic observations are needed for the high $[\mathrm{Mg} / \mathrm{Fe}]$, low velocity dispersion populations.

With the availability of stellar ages from the Gaia mission in the near future, it will become possible to confirm or rule out the currently proposed explanation for the observed chemo-kinematic relations, by requiring that the data are also reproduced by Figure 4. If confirmed by a number of ongoing and forthcoming galactic surveys, our discovery would provide a missing piece in the current understanding of Milky Way disk evolution.

Funding for RAVE has been provided by the Australian Astronomical Observatory; the Leibniz-Institut für Astrophysik Potsdam (AIP); the Australian National University; the Australian Research Council; the French National Research Agency; the German Research Foundation (SPP 1177 and SFB 881); the European Research Council (ERC-StG 240271 galactica); the Istituto Nazionale di Astrofisica at Padova; The Johns Hopkins University; the National Science Foundation of the USA (AST-0908326); the W. M. Keck foundation; the Macquarie University; the Netherlands Research School for Astronomy; the Natural Sciences and Engineering Research Council of Canada; the Slovenian Research Agency; the Swiss National Science Foundation; the Science \& Technology Facilities Council of the UK; Opticon; Strasbourg Observatory; and the Universities of Groningen, Heidelberg, and Sydney. The RAVE Web site is at http://www.rave-survey.org.

\section{REFERENCES}

Abadi, M. G., Navarro, J. F., Steinmetz, M., \& Eke, V. R. 2003, ApJ, 597, 21

Binney, J., Dehnen, W., \& Bertelli, G. 2000, MNRAS, 318, 658

Boeche, C., Chiappini, C., Minchev, I., et al. 2013, A\&A, 553, A19

Boeche, C., Siebert, A., Williams, M., et al. 2011, AJ, 142, 193

Bournaud, F., Elmegreen, B. G., \& Martig, M. 2009, ApJL, 707, L1

Bovy, J., Rix, H.-W., Hogg, D. W., et al. 2012, ApJ, 755, 115

Brook, C. B., Gibson, B. K., Martel, H., \& Kawata, D. 2005, ApJ, 630, 298

Brook, C. B., Stinson, G. S., Gibson, B. K., et al. 2012, MNRAS, 426, 690

de Jong, R. S., Bellido-Tirado, O., Chiappini, C., et al. 2012, Proc. SPIE, 8446, $84460 \mathrm{~T}$

Freeman, K. C. 1991, in Dynamics of Disc Galaxies, ed. B. Sundelius (Göteborgs: Göteborgs University and Chalmers University of Technology), 15

Freeman, K. C. 2010, Galaxies and Their Masks (Berlin: Springer), 319

Gilmore, G., Randich, S., Asplund, M., et al. 2012, Msngr, 147, 25

Hammer, F., Puech, M., Chemin, L., Flores, H., \& Lehnert, M. D. 2007, ApJ, 662,322 
Haywood, M., Di Matteo, P., Lehnert, M., Katz, D., \& Gomez, A. 2013, A\&A, 560, A109

Jenkins, A. 1992, MNRAS, 257, 620

Kordopatis, G., Gilmore, G., Steinmetz, M., et al. 2013, AJ, 146, 134

Lacey, C. G. 1984, MNRAS, 208, 687

Lee, Y. S., Beers, T. C., Allende Prieto, C., et al. 2011, AJ, 141, 90

Liu, C., \& van de Ven, G. 2012, MNRAS, 425, 2144

Loebman, S. R., Roškar, R., Debattista, V. P., et al. 2011, ApJ, 737, 8

Majewski, S. R., Wilson, J. C., Hearty, F., et al. 2010, in IAU Symp. 265, Chemical Abundances in the Universe: Connecting First Stars to Planets, ed.

K. Cunha, M. Spite, \& B. Barbuy (Cambridge: Cambridge Univ. Press), 480 Matteucci, F. 2012, Chemical Evolution of Galaxies (Berlin: Springer)

Meza, A., Navarro, J. F., Abadi, M. G., \& Steinmetz, M. 2005, MNRAS, 359,93

Minchev, I., Chiappini, C., \& Martig, M. 2013, A\&A, 558, A9

Minchev, I., \& Famaey, B. 2010, ApJ, 722, 112

Minchev, I., Famaey, B., Quillen, A. C., et al. 2012a, A\&A, 548, 126
Minchev, I., Famaey, B., Quillen, A. C., et al. 2012b, A\&A, 548, 127

Perryman, M. A. C., de Boer, K. S., Gilmore, G., et al. 2001, A\&A, 369,339

Quinn, P. J., Hernquist, L., \& Fullagar, D. P. 1993, ApJ, 403, 74

Roškar, R., Debattista, V. P., Quinn, T. R., \& Wadsley, J. 2012, MNRAS, 426, 2089

Schönrich, R., \& Binney, J. 2009, MNRAS, 399, 1145

Seabroke, G. M., \& Gilmore, G. 2007, MNRAS, 380, 1348

Sellwood, J. A., \& Binney, J. J. 2002, MNRAS, 336, 785

Siebert, A., Williams, M. E. K., Siviero, A., et al. 2011, AJ, 141, 187

Steinmetz, M., Zwitter, T., Siebert, A., et al. 2006, AJ, 132, 1645

Villalobos, Á., \& Helmi, A. 2008, MNRAS, 391, 1806

Wielen, R. 1977, A\&A, 60, 263

Wyse, R. F. G. 2001, in ASP Conf. Ser. 230, Galaxy Disks and Disk Galaxies, ed. J. G. Funes \& E. M. Corsini (San Francisco, CA: ASP), 71

Yanny, B., Rockosi, C., Newberg, H. J., et al. 2009, AJ, 137, 4377

Zwitter, T., Siebert, A., Munari, U., et al. 2008, AJ, 136, 421 